ACADÉMIE DES SCIENCES

\section{Un mécanisme conservé à la base de la découpe des feuilles}

Thomas Blein

$>$ Les feuilles présentent une grande diversité de formes entre les espèces végétales et parfois même au sein d'une seule plante. Cette variabilité résulte principalement de différences de niveau et d'organisation de la découpe. Cette dernière peut affecter entièrement le limbe, le découpant en différentes unités appelées folioles, formant ainsi des feuilles composées (Figure 1). En l'absence de telles folioles, les feuilles sont dites simples. Les découpes peuvent être limitées au bord des feuilles et selon leur amplitude former des bords lisses ou fortement lobés [1, 2].

\section{Formation des feuilles} et facteurs de découpe Quelle que soit leur forme finale, les feuilles émergent au sein du méristème apical comme de simples structures cylindriques appelées primordia. Contrairement à la majorité des feuilles simples, le développement des feuilles composées requiert le maintien d'une activité organogénique dans leurs primordia. Selon les espèces, deux voies réalisant cette fonction ont été identifiées : la voie des gènes méristématiques knotted-like homebox (KNOX) et celle des gènes méristématiques floraux LEAFY (LFY) [1, 2]. Ces deux voies sont responsables de la formation d'une zone pseudo-méristématique sur les bords des primordia appelée blastozone qui, après croissance et fragmentation, va donner naissance à des primordia de folioles [1, 2]. Le «dissecteur » requis pour cette fragmentation n'est cependant pas connu, et il n'est pas clair s'il

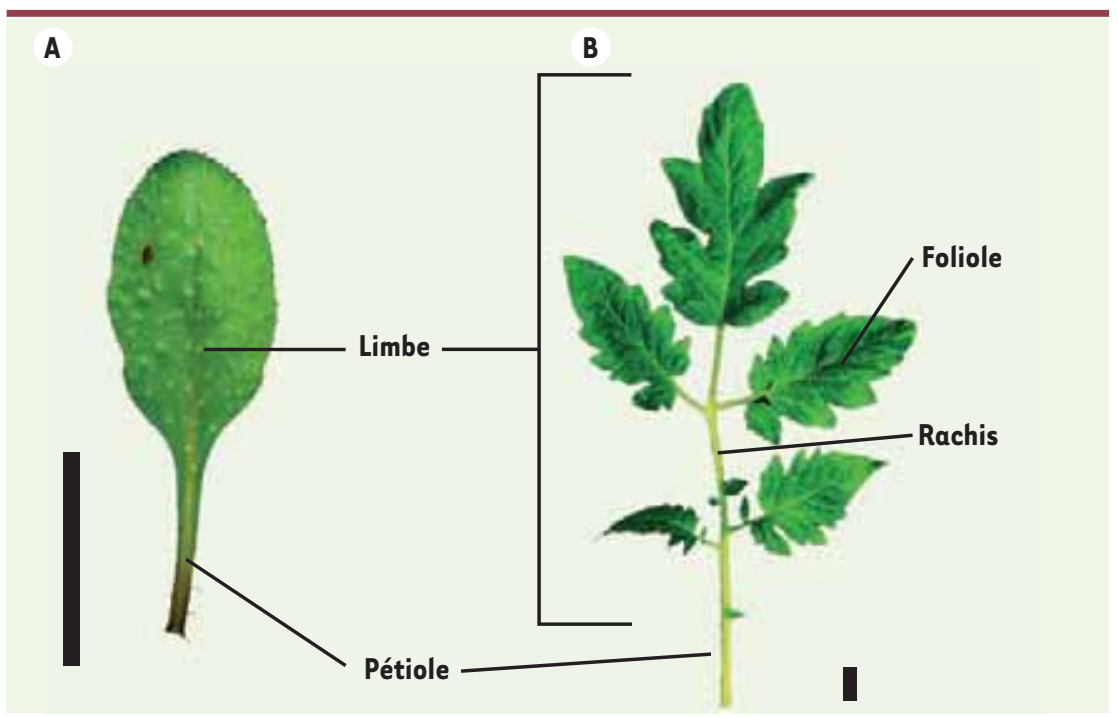

Figure 1. Feuille simple et feuille composée. Les feuilles simples, comme celle d'Arabidopsis (A), sont constituées d'une seule unité de limbe rattachée à la tige par le pétiole. Dans le cas des feuilles composées, comme celle de la tomate (B), le limbe est découpé en plusieurs unités appelées folioles réunies sur le rachis. Les unités de limbes sont plus ou moins découpées avec des bords légèrement dentés comme chez Arabidopsis ou fortement lobés comme chez la tomate (échelle : $1 \mathrm{~cm}$ ).

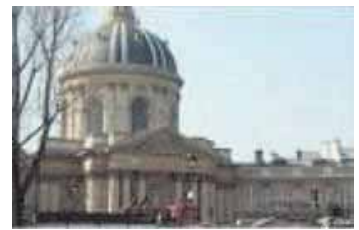

cellulaire,

INRA de

Versailles, RD10, Route de Saint-Cyr,

78026 Versailles Cedex, France.

thomas.blein@biologie.uni-freiburg.de

est conservé entre les espèces à voies KNOX et LFY.

De tels facteurs de découpe ont cependant été identifiés dans d'autres contextes du développement des plantes, comme lors de l'initiation et la séparation des organes. Parmi ceux-ci, on trouve les gènes no apical meristem (NAM) et CUP-SHAPED COTYLEDON (CUC) appartenant à une famille conservée de facteurs de transcription spécifiques des plantes [3-5]. Ces gènes sont exprimés dans les zones frontières entre le méristème et les primordia et contribuent à la faible prolifération cellulaire de ces zones. Leur inactivation entraîne des défauts de séparation d'organes [3-5]. II a également été montré que le gène CUC2 contrôlait la profondeur de la dentelure de la feuille simple d'arabidopsis [6]. Les gènes NAM/CUC, du fait de leur rôle de dissecteurs à la fois dans le méristème et le long du bord de la feuille, sont de bons candidats pour jouer un rôle similaire dans la blastozone. Au sein de l'équipe de Patrick Laufs à L'INRA de Versailles, nous avons donc récemment étudié le rôle des gènes NAM/CUC dans le développement de la feuille de cinq espèces: I'ancolie, la pomme de terre, la tomate, le pois et la cardamine, représentant à la fois les différentes voies contrôlant la forme des feuilles et les principaux groupes d'Eudicotylédones.

\section{Les gènes NAM/CUC dans la formation} des feuilles composées

Onze orthologues des gènes NAM/CUC dans ces cinq espèces ont été clonés et une analyse phylogénétique a permis de les classer en deux clades distincts: le clade NAM qui regroupe l'ensemble des 


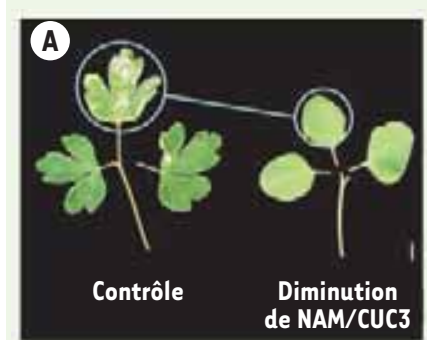

Ancolie

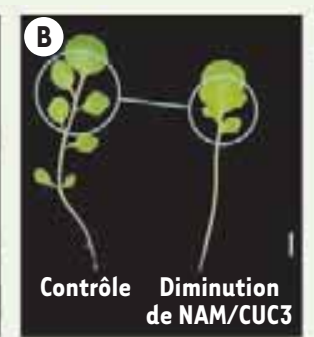

Cardamine

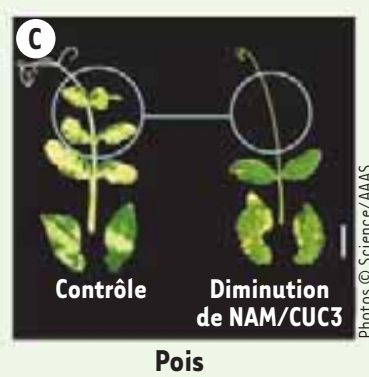

Pois séquences homologues au gène NAM de pétunia ainsi qu'aux gènes $C U C l$ et CUC2 d'arabidopsis [3, 5] et le clade CUC3 qui regroupe les séquences apparentées au gène CUC3 d'arabidopsis [7]. Ces gènes NAM/CUC3 sont tous exprimés dans les frontières, dans un domaine qui est complémentaire aux zones de prolifération, comme les gènes NAM/ CUC3 déjà connus dans d'autres espèces. Au cours du développement des feuilles composées des espèces choisies, ces gènes sont exprimés asymétriquement sur l'un des côtés de la foliole. Cette expression précède l'émergence du primordium de la foliole et reste présente tardivement durant la croissance de la foliole [8].
Ce profil d'expression très spécifique suggère un rôle des gènes NAM/CUC3 dans le développement des feuilles composées que nous avons précisé par une analyse fonctionnelle menée chez quatre des espèces. L'inactivation des gènes NAM/CUC3 a révélé plusieurs de leurs fonctions [4]. Le bord des folioles est dans un premier temps lissé avec la disparition des dents quand elles étaient présentes (Figure 2), révélant ainsi un rôle des gènes NAM/CUC3 comparable à celui observé chez arabidopsis [6]. Des fusions de folioles (Figure 2) indiquent que les gènes NAM/CUC3 participent à la séparation des folioles. Enfin, le nombre de folioles est réduit indiquant qu'en plus de leur

\section{A Développement d'un primordium de feuille composée}

Zone d'expression de NAM/CUC3
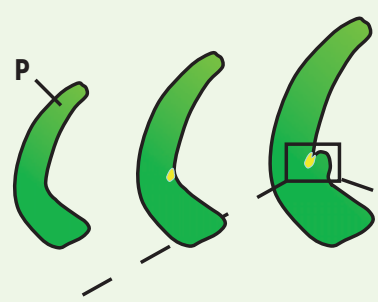
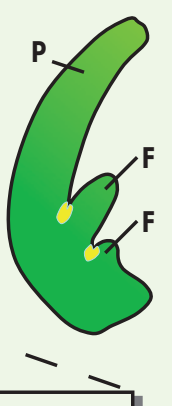

\section{B}

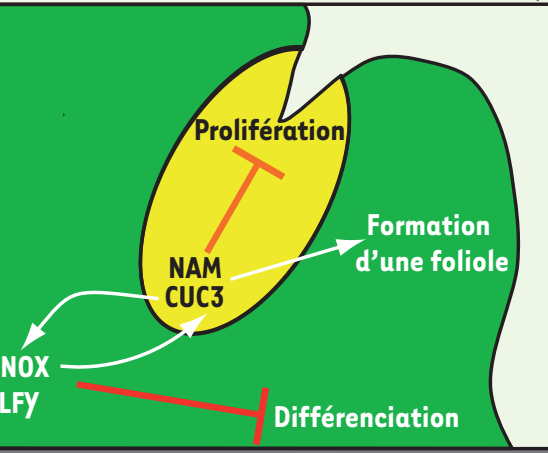

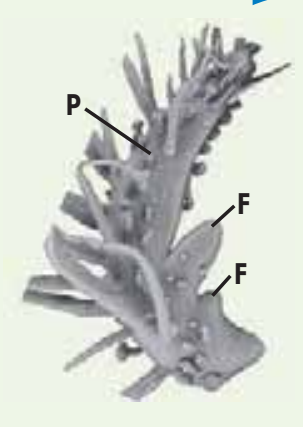

Figure 2. Illustration de la fonction des gènes NAM3/CUC3. L'inactivation des gènes NAM/ CUC3 entraîne un lissage des bords de la feuille (A), des fusions de folioles (B) ainsi qu'une diminution du nombre de folioles (C). Échelle : $1 \mathrm{~cm}$.

rôle dans la séparation des organes, les gènes NAM/CUC3 favoriseraient la croissance des folioles (Figure 2).

\section{Interactions}

entre gènes méristématiques et dissecteurs NAM/CUC

Les interactions pouvant exister entre les gènes NAM/CUC3 et les gènes déjà connus pour leur rôle dans la formation des feuilles composées ont ensuite été étudiées [8]. Dans un premier temps, nous avons montré que les gènes NAM/CUCZ ne sont plus exprimés dans les feuilles simples des mutants Lanceolate $(L a)$ de tomate et unifoliata (uni) de pois. Inversement, l'expression des gènes NAM/CUCZ est augmentée dans une lignée surexprimant un gène de la famille KNOX de la cardamine. Ces résultats suggèrent que les gènes responsables de la découpe des feuilles composées régulent l'expression des gènes NAM/CUC3. Inversement, dans toutes les espèces étudiées, nous avons pu observer qu'une diminution de l'expression des gènes $N A M / C U C 3$, entraîne une forte diminution de l'expression des gènes KNOX et $L F Y$, suggérant ainsi une boucle de rétrocontrôle positive entre ces deux groupes de gènes.

L'ensemble de ces résultats permet de proposer un modèle du rôle des gènes NAM/CUC3 chez les Eudicotylédones

Figure 3. A. L'expression des gènes NAM/CUC3 permet de délimiter les folioles en formation ( $P$ : primordium, $F$ : foliole) $B$. Les gènes NAM/ CUC3 participent à la formation des folioles, d'une part en mettant en place une zone frontière nécessaire à la séparation entre structures voisines et, d'autre part, via leur interrelation avec les gènes KNOX/LFY pour maintenir un état indifférencié. 
(Figure 3). Ces gènes sont exprimés asymétriquement dans la frontière des folioles où ils répriment localement la croissance cellulaire. Ils induisent également la croissance des folioles par un mécanisme qui n'est pas intrinsèque à la cellule et sont impliqués dans une boucle de régulation positive des gènes méristématiques KNOX et LFY. Après avoir identifié ces acteurs conservés de la découpe et de la croissance des folioles, il reste à présent à découvrir les éléments régulant leur activité et ainsi à mieux comprendre l'origine des nombreuses formes de feuilles observées dans la nature. $\diamond$

A conserved mechanism

at the base of leaf dissection

\section{CONFLIT D'INTÉRÊTS}

L'auteur déclare n'avoir aucun conflit d'intérêts concernant les données publiées dans cet article.

\section{Thomas Blein, 28 ans}

Le représentant de la lignée verte a déjà vu beaucoup de choses avant qu'un stage d'été à l'INRA ne le fasse rencontrer les plantes, ayant d'abord parcouru la classe préparatoire de vétérinaire, la faculté et I'INA P-G devenue depuis AgroParisTech qu'il termine avec un Master en biologie végétale. Son travail de thèse qui explore le contrôle des gènes du méristème et de la découpe des feuilles sous la direction de Patrick Laufs à I'Institut Jean-Pierre Bourgin à I'INRA de Versailles. Maintenant en stage postdoctoral dans le département de botanique de l'institut de biologie II de l'université Albert-Ludwig à Fribourg (Allemagne) auprès du professeur Klaus Palme, il reste fidèle à la végétation en s'intéressant désormais à la croissance des racines.

\section{RÉFÉRENCES}

1. Barkoulas M, Galinha C, Grigg SP, Tsiantis M. From genes to shape: regulatory interactions in leaf development. Curr Opin Plant Biol 2007 ; 10 : 660-6.

2. Champagne $C$, Sinha N. Compound leaves : equal to the sum of their parts? Development 2004 ; 131 : 4401-12.

3. Aida M, Ishida T, Fukaki H, et al. Genes involved in organ separation in Arabidopsis : an analysis of the cupshaped cotyledon mutant. Plant Cell 1997 ; 9 : 841-57.

4. Aida M, Tasaka, M. Genetic control of shoot organ boundaries. Curr Opin Plant Biol 2006 ; 9 : 72-7.

5. Souer $\varepsilon$, van Houwelingen $A, K$ loos $D$, et al. The no apical meristem gene of Petunia is required for pattern formation in embryos and flowers and is expressed at meristem and primordia boundaries. Cell 1996 ; 85 : 159-70.

6. Nikovics K, Blein T, Peaucelle A, et al. The balance between the mir164a and cuc2 genes controls leaf margin serration in arabidopsis. Plant Cell 2006; $18: 2929-45$.

7. Vroemen CW, Mordhorst AP, Albrecht C, et al. The CUPSHAPED COTYLEDON3 gene is required for boundary and shoot meristem formation in Arabidopsis. Plant Cell 2003 ; 15 : 1563-77.

8. Blein T, Pulido A, Vialette-Guiraud A, et al. A conserved molecular framework for compound leaf development. Science 2008 ; 322 : $1835-9$

\section{ACADÉMIE DES SCIENCES}

\section{Des progéniteurs transplantés peuvent générer des cellules souches germinales}

Vilma Barroca, Bruno Lassalle, Isabelle Allemand, Lydia Riou, Pierre Fouchet
Laboratoire gamétogenèse, apoptose et génotoxicité,

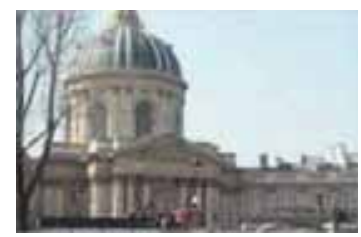
Inserm U967, Institut

de radiobiologie cellulaire et moléculaire, direction des sciences du vivant, CEA, 92265 Fontenay-aux-Roses, France.

vilma.barroca@cea.fr pierre.fouchet@cea.fr
> La spermatogenèse est l'ensemble des divisions et différenciations cellulaires conduisant, à partir des cellules souches germinales (CSG), à la formation des spermatozoïdes dans le testicule adulte. La préservation d'un stock de cellules souches fonctionnelles est indispensable pour le maintien de l'homéostasie et de la fonction tissulaire, mais également pour la régénération du tissu après une lésion. Ces CSG se multiplient soit pour s'autorenouveler soit pour entrer dans le processus de différenciation et donner naissance à des progéniteurs, qui seront à l'origine de la production des cellules spécialisées. Le processus d'engagement d'une cellule souche dans le processus de différenciation est considéré comme irréversible. Ainsi, ces progéniteurs perdent la capacité de régénérer le tissu à long terme. Cependant des études récentes notamment dans le modèle $d u$ tissu germinal chez la drosophile, soulignent la capacité qu'ont des progéniteurs déjà engagés à se reprogrammer et à acquérir à nouveau le potentiel de régénération à long terme des cellules souches [1, 2]. Deux mécanismes pourraient ainsi coexister au sein de certains tissus afin de maintenir le pool de cellules souches: l'autorenouvellement des cellules souches et la reprogrammation des progéniteurs.
Les progéniteurs murins transplantés peuvent régénérer une spermatogenèse à long terme Dans le testicule murin, les CSG ou spermatogonies $A_{s}$ (single) débutent le processus de différenciation pour donner une première série de progéniteurs, les spermatogonies $A_{p r}$ (paired) et $A_{a l}$ (aligned) dites indifférenciées. Les spermatogonies $A_{a l}$ se différencieront en une seconde série de progéniteurs dits différenciés, les spermatogonies $A_{1}, A_{2}, A_{3}, A_{4}$, Int et $B$ dans lesquelles se produira par la suite le processus méiotique (Figure I). Chez la souris, nous disposons d'un test fonctionnel identifiant les CSG: I'analyse 